\title{
THE INVESTIGATION OF HYDROGELS COMPOSITE FILLING BY GELATIN
}

\author{
Chobit M., Panchenko Yu., Vasylyev V.P. \\ Institute of Chemistry and Chemical Technologies, Department of organic chemistry, Lviv Polytechnic \\ National University, UKRAINE, Lviv, 3/4 pl. Sviatoho Yura, e-mail: chobit@ polynet.lviv.ua
}

\begin{abstract}
The paper describes the synthesis and investigation of the properties of acrylamide and acrylic acid hydrogel composites with a crosslinking agent with gelatin, unmodified or modified peroxide oligomer, which can be use to create cosmetic products. The method of synthesis of hydrogel composites described and their physical, chemical, thermomechanical properties and graphic representation of these regularities presented. To do this, a series of samples with different ratios of starting substances synthesized and the dependence of their properties on the structure is established.

Keywords: polymer composite, hydrogels, gelatin, swelling.
\end{abstract}

\section{Introduction}

Hydrogels are one of the perspective classes of polymer systems that embrace numerous biomedical and pharmaceutical applications. Hydrogels have become very popular due to its unique properties such as high water content, softness, elasticity and biocompatibility. Natural and synthetic hydrophilic polymers can be physically or chemically crosslinked to obtain hydrogels. Their resemblance to living tissue opens up many possibilities for applications in biomedical fields. Hydrogels are widely used for various biomedical applications - tissue engineering, molecular imprinting, monopoles as dressings, drug delivery and other.

The aim of the present research is obtaining hydrogel composites filled with gelatin for cosmetic application.

\section{Experimental}

By graft-polymerization of hydrophilic functional monomers in an aqueous medium obtained spatially, crosslinked polymer hydrogels filled with gelatin (Gel). For this purpose, a process conducted in water solution of monomers (acrylamide (АкАм), acrylic acid (AcA)) in presence gelatin and modified gelatin peroxide modifier. In the case of using the modified gelatin of the initiation of polymerization occurred due to the decomposition of peroxide groups immobilized to the surface of the gelatin molecules. The paper presents the evolution of swelling of the gelatin composites with grafted macromolecules of polyacrylamide and polyacrylic acid and different ratios of filler and copolymers.

The degree of swelling (D.S.) was determine by equation is Eq. 1:

$$
\alpha=\frac{m-m_{0}}{m} * 100
$$

$\alpha$ - the degree of swelling \%mas, $\mathrm{m}$ - weight of swollen composite, $g ; \mathrm{m}_{0}$ - the weight of the dry composite, $g$.

Taking into account, the presented results are noticeable sharp increase in the maximum values of swelling in comparison with the samples of grafted polyacrylamide. This is probably due to the formation of an additional stitched mesh between grafted copolymer through centers of interaction between functional groups of macromolecules $-\mathrm{NH}_{2}$ and $-\mathrm{COOH}$. Composites filled with modified gelatin show mass storage, and therefore shape, with long periods in a water environment. This suggests that the modified gelatin has the satisfactory properties of the crosslinked agent.

Figure 1 demonstrated the kinetics of swelling of polymer hydrogel composites based on unmodified gelatin, acrylamide and acrylic acid, which differ in the content of gelatin. 


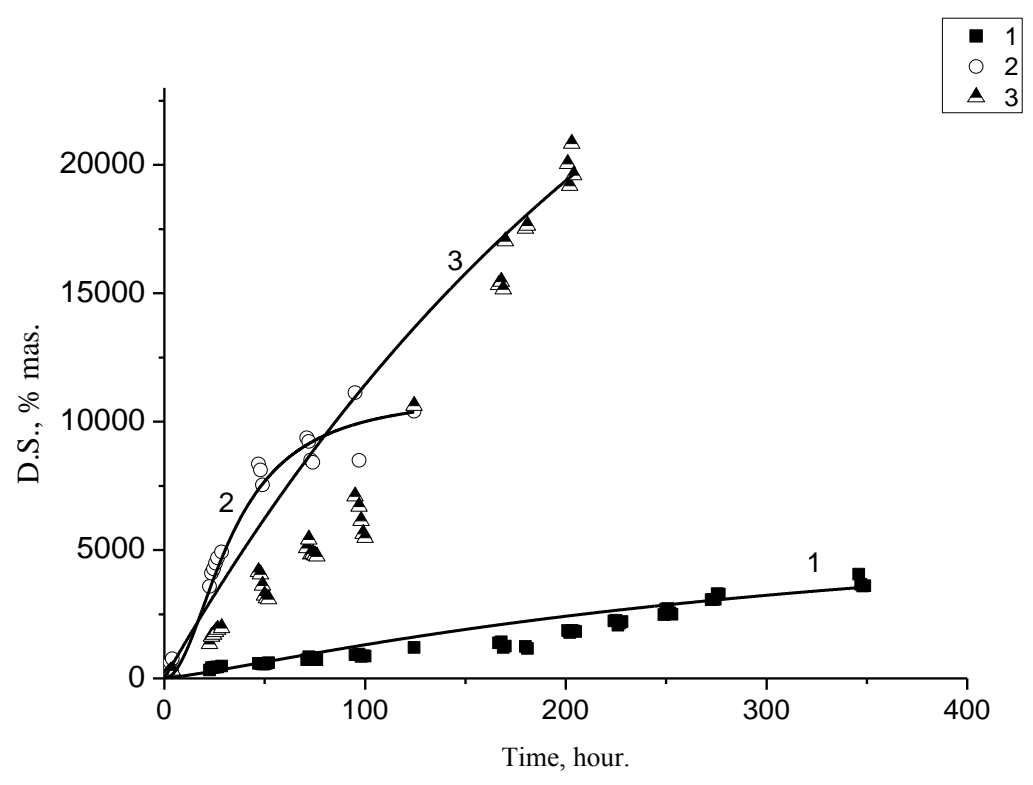

Fig.1. The kinetics of swelling of hydrogel composites.

The composition of the copolymer 1: \%mas: Gel. $=20, \mathrm{A \kappa}_{\mathrm{Am}}=40, \mathrm{AcA}=40$;

The composition of the copolymer 2, \% mas: Gel. $=10, \mathrm{A \kappa}_{\mathrm{M}}=45, \mathrm{AcA}=45$;

The composition of the copolymer 3, \% mas: Gel.=5, АкАм=47,5, AcA=47,5;

The results show that the best sorption properties of the sample has a content of 5\% wt. gelatin. However, the increase of content of gelatin results in the best mechanical durability and possibility of the protracted storage.

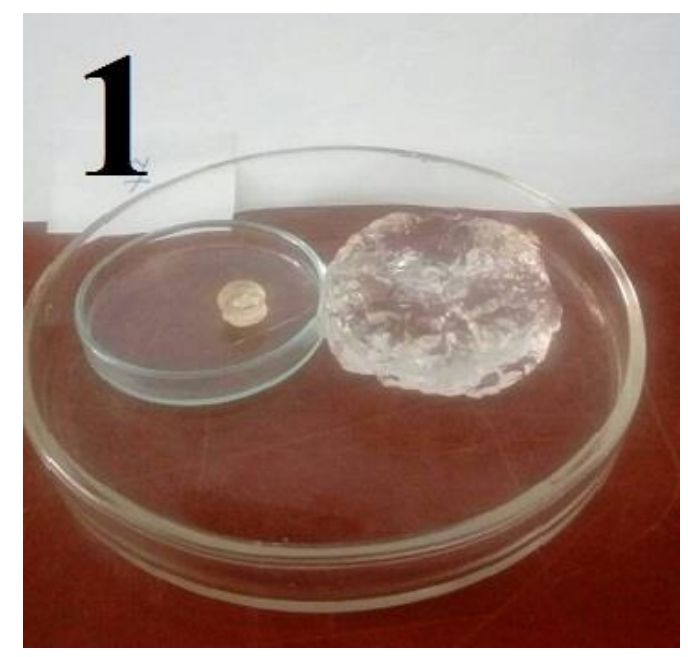

Fig.2. Photo hydrogel composites before and after swelling

\section{Conclusion}

As a result of conducted research, the obtained hydrogel composites with various composition and ratio of components: gelatin (unmodified and modified by the peroxide oligomer of VEP-MA), acrylamide, acrylic acid and starch. The kinetics of swelling of hydrogel composites and determined the rate constants of swelling. The change of sizes of relative change of sizes of the obtained hydrogel composites is calculated and presented. 